\title{
A Siamese Adversarial Anonymizer for Data Minimization in Biometric Applications
}

\author{
Giuseppe Garofalo, Tim Van hamme, Davy Preuveneers and Wouter Joosen \\ imec-DistriNet, KU Leuven \\ Heverlee, Belgium \\ $\{$ name $\} .\{$ surname $\} @$ cs.kuleuven.be
}

\begin{abstract}
A wealth of sensors measure our day to day activities. Sharing these biometric data with cloud-enabled applications provides users with useful insights about their health status. However, due to their high re-identification potential, granting medical and fitness applications access to sensors data raises serious privacy concerns.

In this work, we aim to find a data representation that suppresses identity while retaining utility, i.e. data minimization. To this end, we define a component that is plugged into a machine learning pipeline and trained following a min-max adversarial optimization strategy. By using our component during training, we limit the need to rely on third parties and, therefore, reduce the risks of leakages and unintended processing. We evaluate privacy vs. utility trade-off on a real-world sharing scenario involving motion data, i.e. accelerometer and gyroscope measurements. Privacy is estimated by analyzing if an honest-but-curious attacker can effectively link two traces to the same user. In our experiments, we observe a decrease in test verification accuracy, i.e. the identifiability of the users, from $85 \%$ to $57 \%$. This leaves an attacker with a representation that he can match only slightly better compared to a random guess. On top of that, the privatized gait representation is accompanied by an increase in performance w.r.t. the main tasks.
\end{abstract}

Index Terms-Anonymization, Adversarial Learning, Mobile Sensors, Gait, Soft biometrics

\section{Introduction}

The market for mobile apps in the health and fitness domain has experienced rapid growth over the past years. Undoubtedly, the increasing number of embedded sensors in mobile devices and their enhanced computational resources have played an instrumental role in this expansion. Service providers are now able to implement an array of solutions in the medical area, ranging from fall detection systems [1] to Parkinson's disease predictors [2]. In this scenario, the richness of the captured information is a key factor in developing applications for monitoring patients' health and preventing potentially harmful situations.

This mass collection of highly-sensitive data puts the user's privacy at risk. Moreover, existing cloud-based ecosystems, such as Google Fit APIs [3], facilitate the extracting, managing and storing of mobile sensor data, but do not provide technical protection against misuse of their platform by third parties. The Google Fit APIs documentation articulates the necessity to obtain explicit consent to

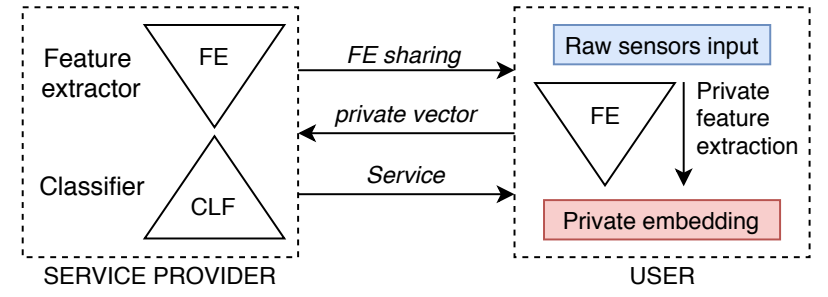

Figure 1: Our framework for private feature sharing, inspired by [8].

access device sensors and encourages developers to rightfully handle users' data. However, there is no technical protection against misuse of the platform by third-parties. As shown by previous studies, this leads to repeated violations against the principles of data minimization and purpose limitation [4]. Additionally, raw motion data from accelerometer and gyroscope measurements is regarded as a behavioural biometric, which embodies the dual nature of biometrics as follows: on the one hand, these measurements can be used to uniquely and accurately identify the user through gait analysis [5], allowing attackers to collude and perform linkage attacks. On the other hand, they incorporate a wide range of sensitive information, ranging from soft biometrics to medical conditions [6], [7], which allows user profiling for purposes beyond the original service, thus yielding function creep.

Legal frameworks are helping to shape the landscape of privacy-preserving biometric data sharing. The General Data Protection Regulation (GDPR) came into effect to raise awareness and encourage the adoption of privacy in the early phases of system design. Biometrics are a special category and require the user to give explicit consent for their processing and storage. However, as mentioned earlier, their dual nature collides with the collection of what is strictly needed for the primary task. This calls for measures to minimize the disclosure of sensitive information while retaining the utility of services offered by, generally untrustworthy, third-parties. We regard data minimization as a fundamental requirement to achieve privacy by design, hence the development of a framework that we refer to as private feature sharing (Fig. 1). Our goal is to deliver a component which accomplishes leakage minimization w.r.t. a private attribute, i.e. the user's identity, on the client side. At training time, this component is designed to extend an existing network for classification without affecting its accuracy (i.e. service utility) nor modifying the original architecture. 
Different approaches exist to minimize leakage while retaining data utility. Randomization-based techniques can be used to protect a user's privacy when sensitive information is shared: they divide into context-free and context-aware methods. Differential Privacy [9] (DP) is the best-known example of context-free techniques. Despite providing statistical guarantees against worst-case adversaries, DP is characterized by a reduced accuracy w.r.t. the target task or by the need to trust a central node for aggregation [9]. In contrast to context-free methods, context-aware techniques define the sensitive leakage as the mutual information between the released data and the private variables [10]. In this way, they tailor the noiseaddition schema to the adversary's capabilities and the desired privacy-utility ratio. Unfortunately, it can be very difficult to formally characterize the relationship between private and public variables due to the highly intertwined nature of the target distributions [10].

This paper overcomes the difficulty of modelling the underlying joint distribution by harnessing a data-driven approach. In fact, it is possible to approximate the mutual information between the primary task and the private task by implementing neural networks in a min-max optimization framework. This process involves three actors: a (private) feature extractor, an (adversarial) discriminator, and a classifier for the target task. The adversarial training procedure shapes the feature encoding function in three iterative steps, as follows: (1) as soon as the input is fed to the extractor, it will try to extract a set of private features; (2) the discriminator challenges the extractor by adapting to its change; (3) by re-training the classifier, high task-specific accuracy is retained. After convergence, the feature extractor is shared with the users while the discriminator is kept private for future use.

This work assesses the retainment of useful information and the removal of potentially identifiable information by performing supervised training [8], [11], [12]. We first show how biometrics features extracted for soft biometrics prediction (e.g. age estimation) can be used for authentication, even though the latter was not initially envisioned by the user. Then, we extract private features from motion data to evaluate the empirical privacy-utility trade-off. To this extent, a new discriminator, i.e. a siamese verifier, and classifier, i.e. a support-vector machine (SVM), are trained on the private features. To further highlight the benefits of adversarial learning, the results are compared to the following approaches: context-free mobile sensors noise addition [13], and multi-objective optimization [8].

In summary, we identify three main contributions.

1) We demonstrate the privacy leakage in a state of the art model for sensitive inference in light of its implementation as a cloud-based solution.

2) We define a pluggable component to achieve data minimization, and incorporate privacy in the design without compromising the primary utility.

3) We devise a novel training strategy for private feature extraction of gait traces.

\section{Related work}

Our goal is to emulate our adversary's capabilities and underlying dataset statistics in a context-aware manner.
This is derived from the inherent difficulty of characterizing the relationship between released data and private variables in the biometric domain, which limits us in the use of formally proven techniques [14].

In order to achieve our goal, we look into recent advances in adversarial learning and generative networks for privacy. Despite being considered difficult to train and tune, these techniques have gained attention in the security community as a means to protect a user's privacy along the whole machine learning pipeline, from the input to the output of the model. Recently, Nasr et al. [15] have exploited adversarial regularization to discourage the model from learning information about the training dataset and achieve membership privacy. In our work, we investigate the application of such min-max optimization strategy by focusing on the latent space, i.e. the learned representation, rather than the output of the classifier.

Edwards et al. [16] first introduced the adversarial competition for learning a private encoding in the visual domain. We adapt their finding to our specific use case: adversarial training for mobile sensor data. It is worth to notice their link to domain-adaptation [17], which has a fundamentally different scope of generalizing the learned function across domains instead of modelling an attacker. Generative adversarial networks (GANs) overcome this limitation, thus they have been recently proposed as an effective tool to achieve context-aware privacy. GANs model a min-max game between a generator and a discriminator, where the former tries to fool the latter in an iterative learning process. This concept has been adapted to the privacy domain first by Huang et al. [10] which define the generative adversarial privacy (GAP) framework. In a similar vein, we obtain a private encoder which outputs a compressed template for a specific, measurable and limited purpose, while also minimizing sensitive disclosure.

A common trait of some GAN-like approaches is the removal of private information from the input by producing a private sample in the original input space [12], [16]. Instead of resembling the original sample, we focus on learning a private, compact representation that just accounts for the information we need. We leverage adversarial training as our selective noise addition strategy. The only similar work is that of Pittalunga et al. [11]. Unlike them, we tailor gait analysis and verification of the user instead of private variables in the visual domain, which has been tackled by several recent works [18]-[20]. Identity privacy in the soft biometric recognition area have been recently tackled, in the visual domain, by Wu et al. [19]. They maintain a high utility for gender, age and race while discarding the user identity, which justifies our learning goal in a completely different domain, i.e. the sensorbased gait domain. In fact, to the best of our knowledge, this is the first work tackling the soft biometrics learning task from an identity-preserving perspective. The use of one of the largest databases also allows us to asses the privacy of our approach from a verification perspective, as can be seen in the visual domain [19].

Our work is comparable with that of Malekzadeh et al. [12]. Similarly, we train a model to be used on the user's device, assigning no trust on third parties, which is something crucial for other works [21]. However, we focus on learning a private encoder as part of the feature extraction step for a specific task. By disregarding 
structural information about the original sample, we reduce the retainment of sensitive information about the user. Moreover, we go beyond the activity recognition task, that has been extensively studied, to tackle a new use case: soft biometrics estimation. The authors train a discriminator which is an authenticator for the 24 users present in their dataset. In order to develop a scalable approach towards classifying the age of the users, we apply siamese networks and a distance-based loss function, i.e. contrastive loss, as our discriminator. This accounts for a more realistic scenario where few and short samples are given from many different users. Finally, we develop our model starting from an existing state of the art pipeline for sensitive inference, providing two additional advantages of (1) estimating the identifiability of the extracted features and (2) deriving a pluggable component to be used in different contexts.

The use of contrastive loss to train the two legs of a siamese model is not new to gait recognition from sensors data [22], [23]. Osia et al. [8] make use of contrastive loss function to enforce identity separation, while retaining useful information. However, they fine-tune part of a pretrained model, while adding noise to achieve their goal. In our work, we optimize the two tasks together, to make the most out of the optimization procedure without the need for a noise addition strategy. Additionally, we argue that reducing the distance between similar traces in the latent space could be much harder in the case of regression, which we evaluate, and multi-class learning. Our loss function does not depend on the primary task, enforcing small distances between different identities instead.

Deep learning for mutual information estimation has been been investigated by Belghazi et al. [24] which define a framework for improving generative networks representation learning. Romanelli et. al [25] compute mutual information exactly to train a generative network with optimal privacy-guarantees. Their method rely on discrete variables and the knowledge of their prior distributions. Differently, we define our privacy in terms of mutual information between two variables but we estimate it through a loss function, since it is hard to model the relationship between the identity or the soft biometric and latent representation extracted from our model.

Random noise addition has been studied as a trivial privacy-preserving technique in the gait domain. Matovu et al. [13] devised a noise injection strategy to be applied on raw data to analyze the impact on the authentication error rate. They concluded that the effectiveness of such techniques is highly application-dependent, making it difficult to perform a reliable and thorough assessment. Another way of performing noise injection is to apply it at the level of the features, as proposed by Osia et al. [8]. They use noisy embeddings to enforce the separation between two feature vectors extracted from the same user. We use these techniques as a baseline for comparison to our private feature extractor in Section 4.

\section{Private feature extraction framework}

In this section, we present our optimization framework in three steps: (1) an overview is given, including our goals and involved actors; (2) the privacy vs. utility trade-off is discussed and formalized as an optimization function; (3) we describe the architecture of the model, which is the realization of the optimization function.

\subsection{Overview}

In our framework, we identify two main actors. The first one is the service provider, which performs data analytics on behalf of its users. The second one is the user himself, who provides data to its counterpart in exchange for some utility. Sharing the raw biometric template, as it is common in mobile health applications, leads to several potential issues. For example, a service provider might infer information that are not strictly required to accomplish the main task, such as whether he suffers of a particular medical condition or not [2]. Hence, the service provider is an honest-but-curious adversary, behaving legitimately but attempting to gain as much knowledge as possible from the received data. This is a plausible assumption given that biometrics embed numerous information that are severely interlaced and, therefore, difficult to tear apart [6].

In this work, we study the inclusion of a privacyminded block in a deep learning model to enhance user's protection with little to no utility loss. We therefore identify data minimization (DM) as a precondition to embody privacy in our workflow. However, when developing privacy-preserving solutions, DM can be regarded as an abstract concept open to interpretation. Following Guersas et al. [26], we identify two relevant challenges to consider for embedding DM in our target application: minimizing the assumptions we put on the provider and minimizing the risks of leaking private information. The former is achieved by keeping private information on the user's device, i.e. sanitizing the input locally. In this way, raw biometric templates never leave the client. The latter requires data to only contain relevant information for the original task, as enforced by the data minimization principle (Article 5 of GDPR). Here, we consider two possible leakages. The first one is the re-identification of the user, i.e. is the attacker able to infer if two traces belong to the same user? The second one is the leakage of side attributes, i.e. is the feature vector minimal w.r.t. the primary task? To summarize, we aim for a solution which is local, pluggable, and minimizes the risks of privacy leakages.

To achieve our goals, we propose a privacy-preserving framework which favours collaboration over trust among the involved entities (Fig. 1). The service provider trains a model in the cloud by including a privacy-preserving block. Training only leverages data that is already accessible to the public. The model can be divided into a feature extractor and a predictor that is trained on the extracted features. After training, the feature extractor outputs features that are (1) minimized w.r.t. the main task and (2) are not linked to the users of the system, who is never involved during training. This allows to publish the feature extractor, e.g. an application downloaded by the users, which extracts features to be sent back to the provider. The output prediction, e.g. medical diagnosis, is eventually shared to the user over a reliable channel. It is worth to notice that publishing a feature extractor has multiple desirable side effects. First, it allows third-parties to audit and verify its privacy, making it hard for thirdparties to collude and turn malicious. Second, it can be 


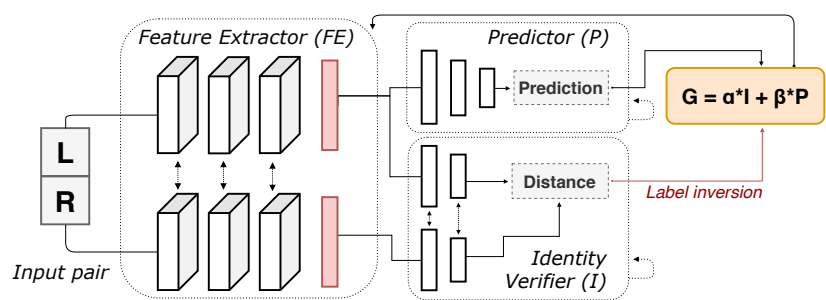

Figure 2: The architecture of our network featuring three main components.

deployed as a lightweight component to be used locally, since it does not involve any training step on the client side. Third, by compressing the initial representation, we discourage communication latency when dealing with multi-dimensional and memory eager input.

\subsection{Optimization function}

We look for the optimal feature extractor $F E($.$) ,$ such that given an input trace $X$ a private embedding $Z=F E(X)$ is obtained. Given that $D(.,$.$) is a measure$ of the dependency between two variables, $D(I, Z)$ measures the dependency between the identity $I$ and the latent representation $Z$, while $D(T, Z)$ measure the dependency between $Z$ and the task we want to preserve. As a result, the nominal loss to be minimized is

$$
L=\alpha * D(I, Z)-\beta * D(T, Z),
$$

where $\alpha$ and $\beta$ regulate the relative importance of each term during training. We approximate the dependency measures by means of a deep neural network architecture which follows an adversarial optimization training.

\subsection{Neural network architecture}

As shown in Fig 2, the network features three subcomponents. The first two elements, a feature extractor (FE) and a task-dependent predictor (P) are inherited from [7], while a third component represents the privatizer, i.e. the siamese identity verifier (I).

We evaluated two scenarios: feeding the model with raw data from the sensor and normalizing the input trace, i.e. scaling the inputs to unit L2-norm. Likewise, normalization is applied to the latent space embedding upon retraining discriminators. This ensures that distance-based loss functions are not affected by the scale of the features, which is especially useful in order not to introduce bias in our evaluation framework since we are going to modify the classification model.

FE is inspired by [7], where the authors demonstrated state of the art performance in soft biometrics prediction from gait traces by using dynamic features. As proposed by Bai et al. [27], we exploit temporal convolutional networks (TCNs) as our feature extraction block. When dealing with time series, TCNs constrain an output at time $t$ to depend only on inputs that have been observed in the past, within the interval $[0, t]$. TCNs use causal convolutions for convolving the output at time $t$ with past input values, up to a specific time instant. To overcome the linear growth of the receptive field, we make use of dilated convolutions, firstly introduced by Oord et al. [28]. They enable the exponential growth of the receptive field by stacking dilation layers. Dilated convolutions account for low-level spatial accuracy as well as a global view of our input because of a large receptive field, while the overall complexity is kept low. It is important to highlight that a low complexity is the catalyst for a mobile implementation of the privatizer.

In order to cope with over-fitting, TCN layers are intertwined with spatial dropout layers. These act as regularizers by zeroing-out randomly-chosen feature planes, or channels, during the training phase. The same channels are re-activated at testing time to unleash the full potential of the network. Following best practices, $L 2$ regularization is introduced to penalize complex models through the loss function. Finally, a global average layer flattens the output of the extractor: 6 instant measurements from the inertial sensor become a single value, for a total of 128 features.

The extracted features are conveyed in two separate networks: $P$ and $V$. These blocks are trained adversarially with $F E$ in an effort to learn meaningful patterns for our target problem while minimizing the retainment of useridentifiable information.

The predictor $P$ models our target goals by predicting the age or the gender of a user. This network is trained to optimize the prediction accuracy, which means minimizing the mean absolute error (MAE) of the predicted age and maximising the classification accuracy of the predicted gender. In practice, these nominal targets are approximated by loss functions when training the neural network. The $M A E$ remains valid for age while the binary cross-entropy is selected for the gender predictor:

$$
H(l, p)=(l * \log (p)+(1-l) \log (1-p)) .
$$

The second block is the siamese identity verifier $I$. The model is composed of two clone branches which share their weights. They take the latent representation of two separate traces as their input and try to learn a new representation which allows for extracting the users' identity. This is achieved by increasing the similarity of pairs based on a distance metric, the Euclidean distance, while simultaneously removing dissimilar pairs. A pair of samples is presented at the input along with a label embodying the similarity notion, i.e. the identity of the users in our case. Eventually, the output of the two branches is combined in the constrastive loss layer, first introduced by Hadsell et al. [29] as the following function:

$$
C L=y * d^{2}+(1-y) * \max (m-d, 0)^{2},
$$

where $d=\left\|f_{1}-f_{2}\right\|_{2}^{2}$ is the Euclidean distance between the feature vectors, $y$ encodes the similarity of the pair with $y \in[0,1]$, and $m$ represents the margin which constrain the separation.

The optimization follows a strict alternation policy, as shown in Alg. 1. At the beginning of each epoch, the training set is shuffled. It is then divided into mini-batches that are used to train every component in isolation: first, $I$ and $P$ are fed with the output of $F E$ and updated according to their respective losses; second, the two networks are frozen, the labels of the pairs are reversed, and the FE is trained on the multi-objective loss function

$$
G(\theta, \phi)=\min _{\theta} \max _{\phi}(\alpha * I(\phi)+\beta * P(\theta))
$$




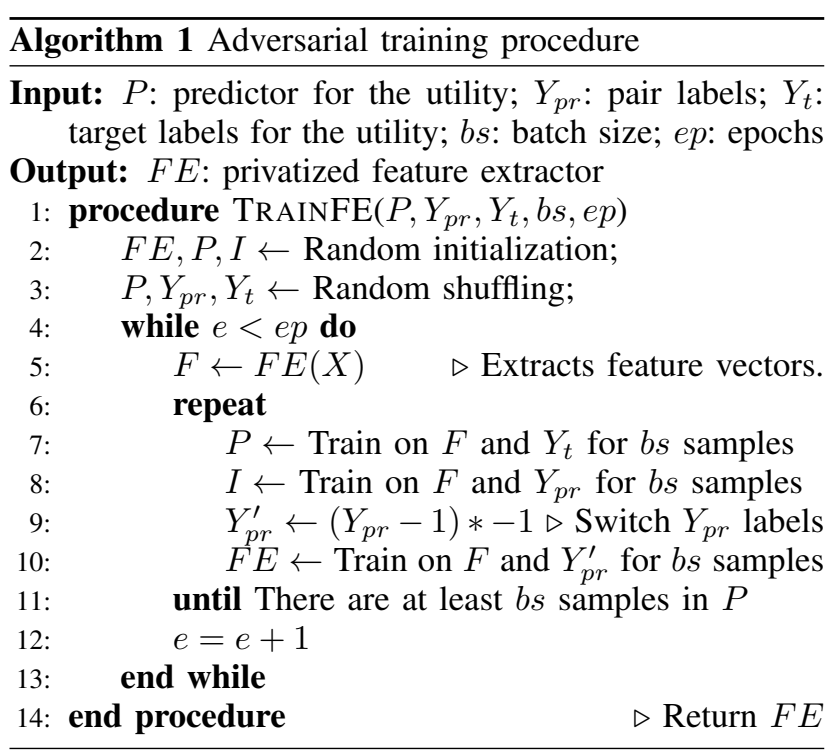

where $\theta$ and $\phi$ are the weights of the predictor $P$ and the verifier $I$ respectively; $\alpha$ and $\beta$ the hyper-parameters weighting the importance of each of the two terms. The network is trained till saturation, i.e. until a sub-optimal solution to the problem is found.

The competition between $I$ and $F E$ is the backbone of privatization: the continuous adaptation of $I$ to the newly learned features, emulating an adversary, prompts the adaptation of FE. This is done by switching the labels of the pairs and, consequently, switching the classes that are approached in the latent space. As a result, similar pairs are detached and dissimilar pairs get close to each other. The competition between the $F E$ and $I$ models the dependency measure of $L$ (Eq. 1) empirically. In order to prove that the final embedding obstruct the reidentification task, the verifier and the classifier are reengineered and re-trained starting from the privatized and non-privatized features.

\section{Evaluation}

In this section, we test our approach to privacypreserving data sharing. To this end, we select the model proposed by Van hamme et al. [7]. This model classifies two sensitive attributes, which are the gender and the age of the user. For both tasks, we identify the identity as the private attribute the user does not want to share with the service provider. Our goal is to re-train the feature extractor to retain the least amount of identifiable information while preserving utility.

Once trained, the feature extractor is set to be shared with the users, therefore tested according to two qualitative metrics: utility and privacy. In the evaluation, we estimate the utility by assessing the accuracy of the main classification task, while privacy is measured as the ability to determine whether two feature vectors belong to the same user or not. Using transfer learning, i.e. freezing the feature extractor, we mimic an honest-but-curious adversary who is willing to expand his knowledge about one of his users. After receiving a vector of features, he will jeopardize the privacy of the users by training a model to exacerbate their identities. This enables us to perform an empirical analysis of the leakage in the original feature space (are the extracted features minimal for the given task?) and to apply the following three defenses: noise addition [13], multi-objective optimization [8], and our adversarial optimization training (see Alg. 1). We eventually show the potential of adversarial learning as a privacy-preserving solution for biometrics.

\subsection{Dataset}

Following [7], the work is evaluated on the OUISIR labeled gait action dataset [30]. This dataset consists of 495 subjects which performed several activities in a predefined path. Subjects wore a belt with 3 IMU sensors: left hip, right hip and back. 3D angular velocity and 3D acceleration are collected at a sampling rate of $100 \mathrm{~Hz}$. Several activities were recorded but we only considered walking traces in our experiments. By extracting whole walking sequences we ascertain that at least one gait cycle is included in each data sample.

Despite data being collected in a constrained lab environment, the OU-ISIR database is the largest inertial gait database w.r.t. the amount of subjects. It features a fair gender distribution among its users and a wide range of ages, going from 7 to 78 years old. The size of this dataset turns out to be particularly suited to siamese network for identity verification. It includes a wide variety of identities, with limited enrollment data per identity. The average length of a walking sequence is $5.2 \mathrm{~s}$ and for the majority of the users 2 sequences are present.

\subsection{Experimental protocol}

In this section, we describe our evaluation strategy. This includes the user selection, the creation of pairs, and how we train and evaluate our model.

A pruning step selects all the users with at least two walking sequences longer than $2.5 \mathrm{~s}$. We end up with 340 users to be used for generating pairs $p$. This procedure takes the first sequence $s_{1}$ of each user and creates two pairs: a pair of similar sequences, i.e. same user labelled with $y_{i d}=1$, and a pair of dissimilar sequences, i.e. different users labelled with $y_{i d}=0$. We randomly select the second sequence $s_{2}$ of the dissimilar pair from the dataset. The pair is feed to the siamese feature extractor but, as shown in Fig. 2, only the first branch contributes to the training of the soft biometric predictor. Thus, each pair is given a second label $y_{t}$, which is the gender or the age of the user from which the first sequence of the pair was selected. The resulting labelled pair will be $\left\{p=\left[s_{1}, s_{2}\right], y_{i d}, y_{t}\right\}$.

Users are split into training and test sets in a 80\%-20\% ratio. The training set is further split into training $(80 \%)$ and validation $(20 \%)$ sets to prevent over-fitting the former. This split is kept fixed across our experiments, even when re-training the predictor and the siamese verifier. A private set of users, i.e. the test set, is used to assess the generalizability of the approach. In our threat model, the service provider trains the model without knowing or adapting to its future customers.

The training procedure adapts to the defense we decide to adopt and to the target classification task. To obtain 


\begin{tabular}{|c|c|c|}
\hline Task & Utility (Predictor) & Privacy (Verifier) \\
\hline Gender & $74.98 \%$ & $85.78 \% \pm 1.58$ \\
\hline Age & 9.38 years & $74.01 \% \pm 1.98$ \\
\hline
\end{tabular}

TABLE 1: Baseline feature extractor evaluation. The prediction metrics are the f1-score (gender) and MAE (age).

a private feature extractor, we derive three learning procedures which build on top of our baseline model [7]. The extracted features will serve as a basis to discuss the leakage of identity.

As a first defense strategy, we obfuscate the input to the baseline model. We perform noise addition offline to emulate its injection at the sensor level. As pointed out by Matovu et al. [13], the only noise addition strategy which prevents signal reconstruction while maintaining utility is based on a uniform distribution. Thus, we select the minimum and maximum values for each input channel in the training set, and we draw 6 samples uniformly. Given a variable length gait signal, noise is added individually. An hyper-parameter is introduced as scaling factor: noise_level $\in[0.1,0.2,0.3,0.4,0.5]$

A second solution consists of performing multiobjective optimization, as proposed by Osia et al. [8]. This techniques aims at considering the variable we want to hide while also optimizing the result for the main task. Hence, we plug the privatization block in the original architecture and we train the whole model to minimize $G$ (Eq. 4). The number of epochs is fixed to 300 and an early stop condition monitors the validation loss of the main task up to 20 epochs without a relevant improve. By associating an early stop condition to the validation loss of the main task, we ensure that the validation accuracy is maximized and the verifier does not take over the predictor.

A third, and last, approach is our adversarial learning strategy. We plug the privatization block as before, but we train it respecting the strict alternation policy presented in Alg. 1. The network is trained till saturation, with a number of epochs $e p=300$. This is justified by the empirical results, showing the need for the accuracy to first diminish in order to achieve an acceptable sub-optimal solution. All the approaches leverage the Adam optimizer with a fixed learning rate equal to $10^{-5}$.

As is common in related work, we measure the retainment of identifiable information via transfer learning [8], [11]. First, the test set is presented at the input of the extractor to obtain the feature vectors. Then, a siamese identity verifier is trained from scratch on the private features, trying to learn a new encoding which maximizes the verification accuracy. We make sure that no model-related bias is introduced by re-tuning the number of nodes of the hidden layers, so that the final solution is independent by the model used to train the feature extractor. It is worth to notice that more powerful networks might lead to an improved accuracy, but non-linear classification represents a better proxy for mutual information estimation than only proving linear separability between two representations.

With respect to the evaluation of the accuracy of the main task, we move from a deep network to a traditional ML model. A support vector machine (SVM) is trained to estimate the gender and a support vector regressor (SVR) is fitted to estimate the age. An exhaustive search is run

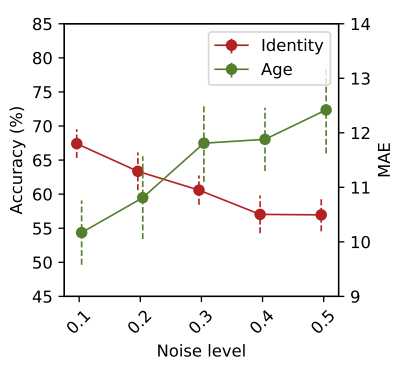

(a)

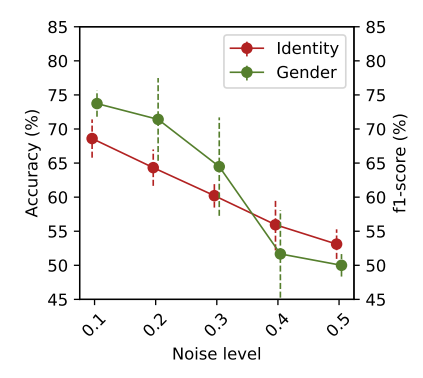

(b)
Figure 3: Uniform noise addition strategy for (a) age and (b) gender. Note that a high MAE means low utility.

on a wide range of hyper-parameters to obtain the best estimator. Eventually, the test set is used to evaluate the obtained models.

We repeat each experiment 10 times, reporting the mean absolute error (MAE) for the age and the average f1-score for the gender. Notably, the privacy is estimated as the verification accuracy, i.e. a high value corresponds to low privacy. Differently, the utility depends on the chosen task: a high MAE means a low utility for the age, while a high prediction accuracy represents a high utility for gender prediction. Excluding the f1-score and MAE that result from a deterministic search, the results are accompanied by their standard deviation.

\subsection{Experimental results}

As a baseline, we train the network as in [5] by setting $\alpha=0$ in $G$ (Eq. 4). Table 1 reports the privacy and utility metrics for the extracted features. As expected, the predictor behaves comparably to the model we took inspiration from, presenting a $75 \%$ accuracy for gender prediction and a average error of 9.38 years for age estimation. In order to verify the retainment of identifiable information, we further train a siamese identity verifier on the extracted features. Despite the features were optimized for the main learning task, the empirical evidence is compelling. The verification accuracy in the age case is $74 \%$, going up to $85 \%$ in the age case.

Fig. 3 shows the privacy and the utility as a function of noise_level. As expected, we observe a dip in the verification accuracy (Fig. 3a) as the noise_level increases. However, this is associated to an important increase in the MAE, which leads to a poor privacy-utility tradeoff when noise_level $>0.1$. A similar behaviour is observed with gender prediction (Fig. 3b). The privacy of the features grows but the performance of the classifier makes the features useless for noise_level $>0.3$. Overall, the gender features are more resilient to moderate noise injection compared to the age features.

Next, we train the network as a whole to emulate the multi-objective optimization defense strategy. The privatization block is now incorporated in the network and its importance is refined by tuning $\alpha$ in $G$ (Eq. 4). In this case, the verification accuracy is not particularly affected by the procedure, keeping the identifiability of the features high. On the other hand, During training, we observe a verification accuracy of the validation set which is constant at $50 \%$. This means that the identity verifier finds 


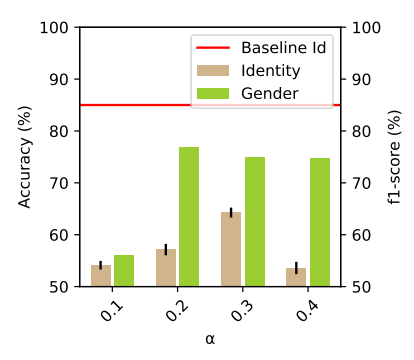

(a)

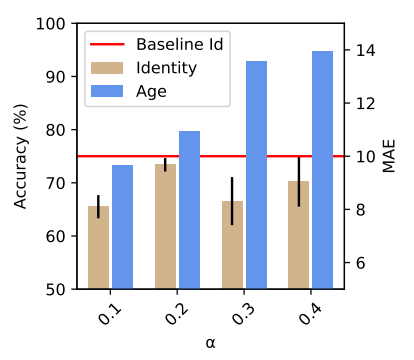

(b)
Figure 4: Privacy vs. utility trade-off w.r.t. $\alpha$ for (a) gender and (b) age.

a sub-optimal solution that the feature extractor can easily fool. However, by simply re-training the identity verifier, we notice that the identity of the user is retained by the learned features, up to $85 \%$ in the worst case.

As a final line of defense, our adversarial optimization strategy is tested (Fig. 4). Beyond $\alpha$, batch_size is also considered and tuned empirically, i.e. only the best setting is shown. Fig. 4a shows the average verification accuracy and the f1-score w.r.t. $\alpha$ for batch_size $=15$. A clear decrease in verification accuracy is visible. Interestingly, the relationship between $\alpha$ and the identity verifier is not linear, and the best trade-off between privacy and utility is found when $\alpha=0.2$. In this case, we notice an increase in the f1-score of the predictor w.r.t. to our baseline, while the average verification accuracy drops from $85 \%$ (Baseline $I d$ ) to $57 \%$. This significant decrease suggests that, given a labelled set of feature pairs, it is almost impossible for an attacker to confidently determine if two feature vectors belong to the same user. Hence, we obtain data minimization w.r.t. the private and main tasks.

Fig. $4 \mathrm{~b}$ plots the average verification accuracy and MAE w.r.t $\alpha$ for batch_size $=25$. As one would expect, utility decreases as alpha grows. Despite being less effective than in the gender case, we register a $10 \%$ drop in the verification accuracy up to $65 \%$ for $\alpha=0.1$. Meanwhile, the utility is maintained at a MAE of around 9.5 years.

\section{Discussion}

A discussion of our results, with implications and future improvements, is given in this section.

We proposed a framework for privacy-preserving data publishing of biometrics. Our preliminary results hint at a minimization of the amount of information embedded in the extracted features, in line with the principles of data minimization and purpose limitation. By just adding a privatization block, i.e. the siamese verifier, we embedded the notion of privacy in an existing pipeline. Our use cases, age and gender estimation, are justified by current research in the visual domain and by the use of a state-of-the-art model for soft biometric prediction.

A comparison has been presented between three lines of defense, confirming that adversarial training can effectively hide identifiable information from gait traces. By comparing to multi-objective optimization, we have shown the benefits of continuously adapting your adversary. Our proposed loss function, in the adversarial training framework, reached the best trade-off between privacy and utility in every experimental setup. However, continuously sharing privatized features might threaten the anonymity of the users. Multiple traces retain minimal information that adds up with time, eventually leading to a unique fingerprint of the user. Moreover, using the same input towards different services can lead to linkage attacks. A possible mitigation is to opt for a blended system leveraging noise addition in conjunction with adversarial optimization. We leave experimentation on the unlinkability of multiple privatized traces to future work.

We tested the removal of the identity of the user from motion data. This can be easily inferred in the gender estimation case, and, to a lower extent, in the age case. Further investigation is needed to understand how and if we can improve the privacy-utility trade-off in the age case to achieve comparable results w.r.t gender. For example, a more efficient search strategy for the hyper-parameters, e.g. by harnessing Bayesian optimization, could be beneficial. Going beyond age and gender, our analysis can be extended to different services. For example, we might want to analyze the leakage of age, gender, and identity in a system that primarily classifies the ethnicity of a user. By privatizing one of the leaked attributes, and observing the remaining ones, we can characterize data minimization across several sensitive variables. Unfortunately, labels for such variables are lacking in existing datasets, consequently driving the scarcity of empirical evidence in the gait domain. Additionally, since static (i.e. statistical) features are still greatly used in the gait domain, an interesting path is to analyze the privacy implications of using neural networks and its impact on the interpretability of the extracted features.

Two limitations affect our work and they are linked to the dataset being used and the lack of formal privacy guarantees. The first limitation lies in the chosen input, the OU-ISIR dataset. In order to asses the feasibility of the approach in a real world scenario, we would need data sampled from mobile devices in an unconstrained environment. In practice, there is no public dataset with such features, and dataset collected from mobile devices suffer from shortage of users and constrained measurements conditions. The second limitation is tied to our data-driven approach, since we do not provide any formal proofs for privacy, in line with related work [8], [11], [12]. This is due to the relationship between released and private attributes, which is hard to formally characterize. However, the aim of this work is not to provide theoretical privacy guarantees, rather to show the identity leakage a user is subject to and to proactively adapt to minimize the risks by means of a data-driven strategy. Thus, we leave theoretical proofs involving techniques from domain-adaptation [17] and frameworks for mutual information estimation [24] to future work leading in this direction.

\section{Conclusion}

In this work, we presented a novel technique for private feature extraction. We applied this technique on a soft biometric inference use case involving gait data where the goal was to hide the identity of the user. By training the existing model with an additional block in an adversarial framework, we end up with a feature 
extractor that can be re-used on previously unseen users to minimize their sensitive data. Our study is supported by the results that show a dip in the verification accuracy from $85 \%$ to $57 \%$ in best scenario, while slightly improving prediction accuracy. Thereby effectively reducing the certainty whereby two traces can be linked together to a near random guess, i.e. an accuracy of $50 \%$. This demonstrates that data minimization is not only desirable but also possible. We advocate for new techniques to be devised such that the user can feel in control of his own biometric trace, protected against sensitive inferences.

\section{Acknowledgment}

This research is partially funded by the Research Fund KU Leuven. We gratefully acknowledge the support of NVIDIA Corporation with the donation of the Titan V GPU used for this research.

\section{References}

[1] S. Abbate, M. Avvenuti, F. Bonatesta, G. Cola, P. Corsini, and A. Vecchio, "A smartphone-based fall detection system," Pervasive and Mobile Computing, vol. 8, no. 6, pp. 883 - 899, 2012, special Issue on Pervasive Healthcare.

[2] C. Caramia, D. Torricelli, M. Schmid, A. Muñoz-Gonzalez, J. Gonzalez-Vargas, F. Grandas, and J. L. Pons, "Imu-based classification of parkinson's disease from gait: A sensitivity analysis on sensor location and feature selection," IEEE Journal of Biomedical and Health Informatics, vol. 22, no. 6, pp. 1765-1774, Nov 2018.

[3] Google Inc., "Google Fit: Platform Overview," https://developers google.com/fit/overview, accessed 24 February 2020.

[4] A. Papageorgiou, M. Strigkos, E. Politou, E. Alepis, A. Solanas, and C. Patsakis, "Security and privacy analysis of mobile health applications: The alarming state of practice," IEEE Access, vol. 6, pp. 9390-9403, 2018.

[5] T. Van hamme, D. Preuveneers, and W. Joosen, "Improving resilience of behaviometric based continuous authentication with multiple accelerometers," in Data and Applications Security and Privacy XXXI - 31st Annual IFIP WG 11.3 Conference, DBSec 2017, Philadelphia, PA, USA, Proceedings, 2017, pp. 473-485.

[6] A. Dantcheva, P. Elia, and A. Ross, "What else does your biometric data reveal? a survey on soft biometrics," IEEE Transactions on Information Forensics and Security, vol. 11, no. 3, 2016.

[7] T. Van hamme, G. Garofalo, E. Argones Rúa, D. Preuveneers, and W. Joosen, "A systematic comparison of age and gender prediction on imu sensor-based gait traces," Sensors, vol. 19, no. 13, 2019.

[8] S. A. Osia, A. S. Shamsabadi, S. Sajadmanesh, A. Taheri, K. Katevas, H. R. Rabiee, N. D. Lane, and H. Haddadi, "A hybrid deep learning architecture for privacy-preserving mobile analytics," IEEE Internet of Things Journal, pp. 1-1, 2020.

[9] C. Dwork, "Differential privacy: A survey of results," in Proceedings of the 5th International Conference on Theory and Applications of Models of Computation, ser. TAMC'08. Berlin, Heidelberg: Springer-Verlag, 2008, pp. 1-19.

[10] C. Huang, P. Kairouz, X. Chen, L. Sankar, and R. Rajagopal, "Context-aware generative adversarial privacy," Entropy, vol. 19, no. $12,2017$.

[11] F. Pittaluga, S. Koppal, and A. Chakrabarti, "Learning privacy preserving encodings through adversarial training," in 2019 IEEE Winter Conference on Applications of Computer Vision (WACV), Jan 2019, pp. 791-799.

[12] M. Malekzadeh, R. G. Clegg, A. Cavallaro, and H. Haddadi, "Mobile sensor data anonymization," in Proceedings of the International Conference on Internet of Things Design and Implementation, ser. IoTDI '19. New York, NY, USA: Association for Computing Machinery, 2019, p. 49-58.
[13] R. Matovu, A. Serwadda, D. Irakiza, and I. Griswold-Steiner, "Jekyll and hyde: On the double-faced nature of smart-phone sensor noise injection," in 2018 International Conference of the Biometrics Special Interest Group, BIOSIG 2018, Darmstadt, Germany, September 26-28, 2018, ser. LNI, vol. P-282, 2018, pp. 1-6.

[14] J. Wang, S. Liu, and Y. Li, "A review of differential privacy in individual data release," International Journal of Distributed Sensor Networks, vol. 11, no. 10, p. 259682, 2015.

[15] M. Nasr, R. Shokri, and A. Houmansadr, "Machine learning with membership privacy using adversarial regularization," in Proceedings of the 2018 ACM SIGSAC Conference on Computer and Communications Security, ser. CCS '18. New York, NY, USA: ACM, 2018, pp. 634-646.

[16] H. Edwards and A. J. Storkey, "Censoring representations with an adversary," in 4th International Conference on Learning Representations, ICLR 2016, San Juan, Puerto Rico, May 2-4, 2016, Conference Track Proceedings, 2016.

[17] Y. Ganin, E. Ustinova, H. Ajakan, P. Germain, H. Larochelle, F. Laviolette, M. Marchand, and V. Lempitsky, "Domainadversarial training of neural networks," J. Mach. Learn. Res., vol. 17, no. 1, p. 2096-2030, Jan. 2016.

[18] V. Mirjalili, S. Raschka, A. Namboodiri, and A. Ross, "Semiadversarial networks: Convolutional autoencoders for imparting privacy to face images," in 2018 International Conference on Biometrics (ICB), Feb 2018, pp. 82-89.

[19] Y. Wu, F. Yang, and H. Ling, "Privacy-protective-gan for face deidentification,” J. Comput. Sci. Technol., vol. 34, pp. 47-60, 2019.

[20] C. Feutry, P. Piantanida, Y. Bengio, and P. Duhamel, "Learning Anonymized Representations with Adversarial Neural Networks," arXiv e-prints, p. arXiv:1802.09386, Feb 2018.

[21] S. A. Osia, A. Taheri, A. S. Shamsabadi, K. Katevas, H. Haddadi, and H. R. Rabiee, "Deep private-feature extraction," IEEE Transactions on Knowledge and Data Engineering, vol. 32, no. 1, pp. 54-66, Jan 2020.

[22] M. P. n. Centeno, Y. Guan, and A. van Moorsel, "Mobile based continuous authentication using deep features," in Proceedings of the 2Nd International Workshop on Embedded and Mobile Deep Learning. New York, NY, USA: ACM, 2018, pp. 19-24.

[23] D. Deb, A. Ross, A. K. Jain, K. Prakah-Asante, and K. V. Prasad, "Actions speak louder than (pass)words: Passive authentication of smartphone* users via deep temporal features," in 2019 International Conference on Biometrics (ICB), June 2019, pp. 1-8.

[24] M. I. Belghazi, A. Baratin, S. Rajeshwar, S. Ozair, Y. Bengio, A. Courville, and D. Hjelm, "Mutual information neural estimation," in Proceedings of the 35th International Conference on Machine Learning, ser. Proceedings of Machine Learning Research, J. Dy and A. Krause, Eds., vol. 80. Stockholmsmässan, Stockholm Sweden: PMLR, 10-15 Jul 2018, pp. 531-540.

[25] M. Romanelli, C. Palamidessi, and K. Chatzikokolakis, "Generating optimal privacy-protection mechanisms via machine learning,' CoRR, vol. abs/1904.01059, 2019.

[26] S. Guerses, C. Troncoso, and C. Diaz, "Engineering privacy by design reloaded," in Amsterdam Privacy Conference, 2015.

[27] S. Bai, J. Z. Kolter, and V. Koltun, "An empirical evaluation of generic convolutional and recurrent networks for sequence modeling," CoRR, vol. abs/1803.01271, 2018.

[28] A. van den Oord, S. Dieleman, H. Zen, K. Simonyan, O. Vinyals, A. Graves, N. Kalchbrenner, A. W. Senior, and K. Kavukcuoglu, "Wavenet: A generative model for raw audio," in SSW. ISCA, 2016, p. 125.

[29] R. Hadsell, S. Chopra, and Y. LeCun, "Dimensionality reduction by learning an invariant mapping," in Proceedings of the 2006 IEEE Computer Society Conference on Computer Vision and Pattern Recognition - Volume 2, ser. CVPR '06. Washington, DC, USA: IEEE Computer Society, 2006, pp. 1735-1742.

[30] T. T. Ngo, Y. Makihara, H. Nagahara, Y. Mukaigawa, and Y. Yagi, "Similar gait action recognition using an inertial sensor," Pattern Recognition, vol. 48, no. 4, pp. 1289 - 1301, 2015. 